\title{
Application of Tessellation in Architectural Geometry Design
}

\author{
Wei CHANG \\ School of Civil Engineering, Tangshan College, Tangshan, Hebei 063000, China
}

\begin{abstract}
Tessellation plays a significant role in architectural geometry design, which is widely used both through history of architecture and in modern architectural design with the help of computer technology. Tessellation has been found since the birth of civilization. In terms of dimensions, there are two- dimensional tessellations and three-dimensional tessellations; in terms of symmetry, there are periodic tessellations and aperiodic tessellations. Besides, some special types of tessellations such as Voronoi Tessellation and Delaunay Triangles are also included. Both Geometry and Crystallography, the latter of which is the basic theory of three-dimensional tessellations, need to be studied. In history, tessellation was applied into skins or decorations in architecture. The development of Computer technology enables tessellation to be more powerful, as seen in surface control, surface display and structure design, etc. Therefore, research on the application of tessellation in architectural geometry design is of great necessity in architecture studies.
\end{abstract}

\section{Introduction}

Architectural geometry design is an important aspect of architectural design from ancient times to the present. Tessellation, or, Tilling, has been widely used in the field of architectural geometry design, which mostly deals with topological and fractals. A tessellation of a flat surface is the tiling of a plane using one or more geometric shapes (tiles), with no overlaps and no gaps ${ }^{1}$. Also, tessellations can be generalized to higher dimensions and a variety of geometries. There are many types of tessellations, among which the most basic and common ones are grid, hexagon tessellation, triangle tessellation, Penrose tessellation, Kelvin tessellation and so on. It has many advantages: figures in tessellations seem simple, but it can be shaped into extremely complex graphics; tessellation generates according to precise rules and orders, conducive to the standardization of production components; with spatial tessellation design, load distribution can be more reasonable in structure, and the damage of single component will not lead to the collapse of the whole structure; besides, tessellation gets strong adaptability to be connected into various irregular shapes.
Tessellation is of great significance to contemporary architectural geometry design. How this ancient art can solve the present problems in architectural design, and how it can inspire architects to create more rational and complicated forms should be studied and stressed, and space in architecture can be well understood through this procedure. This paper will summarize mathematical rules in tessellations, present the application of tessellation in architecture through history, analyze typical cases in contemporary architectural design with application of tessellation, and explore the feasibility and significance of application of tessellation in architectural geometry design.

\section{Rules of Tessellation}

Defined as tilling of a plane or of space with on overlaps and no gaps, tessellations can be classified as blow:

\subsection{Two-Dimensional Tessellation}

Tessellation in two-dimensional space refers to the tessellation of a plane or of two-dimensional surfaces, and can be simplified into some basic geometries, which are called "prototypes".

\footnotetext{
${ }^{1}$ https://en.wikipedia.org/wiki/Tessellation

*Corresponding author: Email: successC_W@163.com
} 


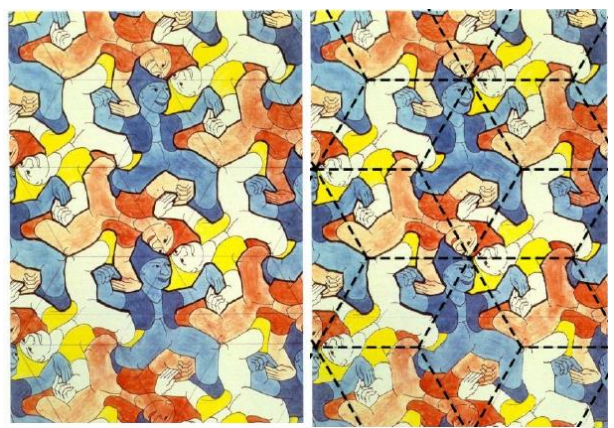

Figure 1 Parts of M. C. Escher Pictures and Its Tessellation

\subsubsection{Symmetry and periodic tessellation}

Many basic tessellations have the attributes of certain symmetries. Symmetry refers to the phenomenon when under some transformation conditions (e.g., around the line for plane rotation, reflection, etc.), an object or a figure in the same part are regular repeated. The symmetry modes of tessellations include translation symmetry, rotation symmetry, reflection symmetry and sliding reflection symmetry ${ }^{2}$.

Tessellation with translational symmetry is called periodic tessellation, such as the regular hexagon tessellation. Periodic tessellation can be represented by lattice, with simple expressions: tessellation systems = elements * lattices. In addition to the common attribute of translational symmetry, plane periodic tessellation tends to have attributes of rotational symmetry, reaction symmetry sliding symmetry.

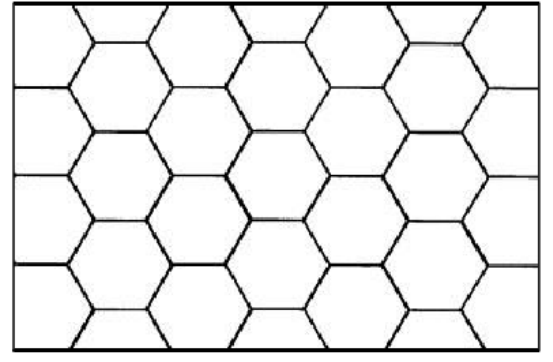

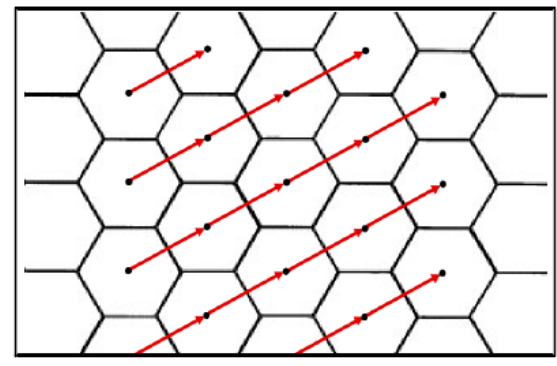

Figure 2 Translational Symmetry Attribute of Regular Hexagon Tessellation

\subsubsection{Aperiodic Tessellation}

Aperiodic Tessellation refers to the translational tessellation without translational symmetry. As shown in

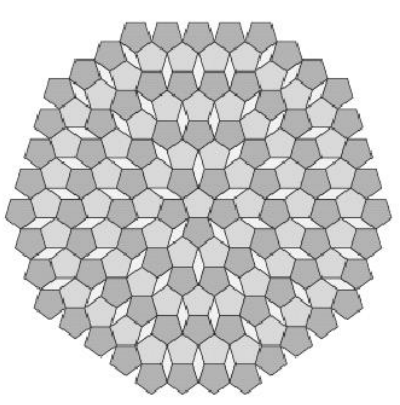

a
Figure3, the figure in the tessellation has rotationally symmetry but no translational symmetry. Similar tessellations include Penrose aperiodic tessellation, Pinwheel aperiodic tessellation, Robinson aperiodic tessellation and so on.

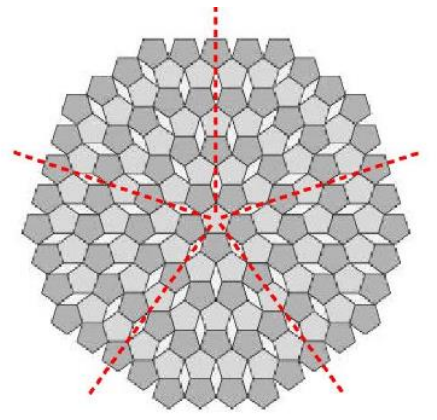

b

Figure 3 Example of Aperiodic Tessellation

\subsubsection{Fractal Tessellation}

Fractals and tessellations are generally considered as two
2 Branko Grunbaum, Tilings and Patterns. W. H.

Freeman, 1987, p26.

*Corresponding author: Email: successC_W@163.com different fields of mathematics, but they can be combined to form a variety of forms with visually attractivities, concluding both fractal and tessellation features. Such form is called fractal tessellation ${ }^{3}$.

\footnotetext{
3 Robert W. Fathauer, Fractal tilings base on v-shaped prototiles, Computers \& Graphics 26, 2002, p635-643.
} 


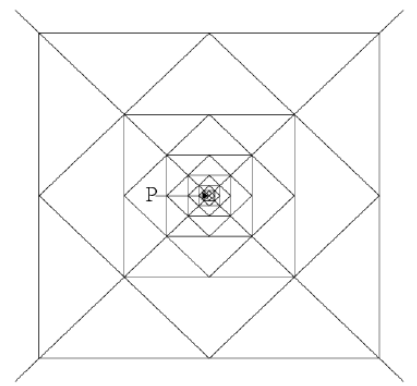

Figure 4 Fractal Tessellation with Isosceles Right Triangle

\subsubsection{Radial Tessellation and Spiral Tessellation}

Another way to create aperiodic tessellation is to select

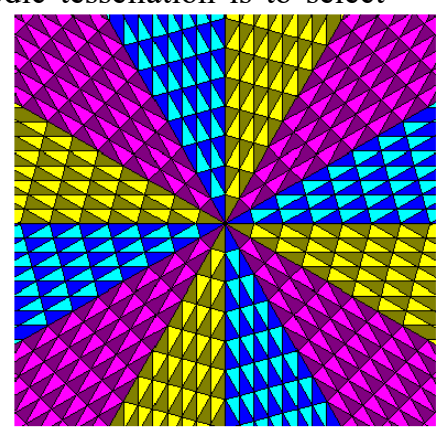

prototypes which can form concentric circles. Such tessellation is called radial tessellation. If parts of the radial tessellation are shifted, a swirl of new type of tessellation can be produced, as called a spiral tessellation.

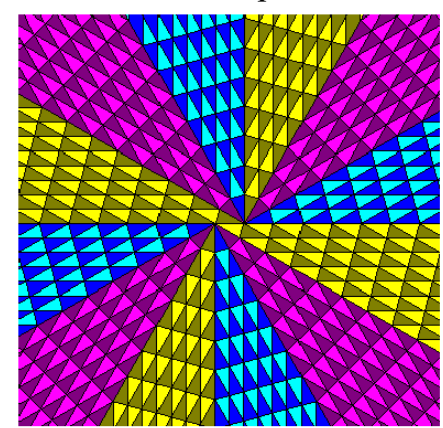

Figure 5 Radial Tessellation and Spiral Tessellation with Isosceles Triangle

\subsection{Three-Dimensional Tessellation}

Three-dimensional tessellation, also called spatial tessellation, has more dimensional variations than twodimensional ones, thus generating more types of geometries.

\subsubsection{Crystal structure and Quasicrystal structure}
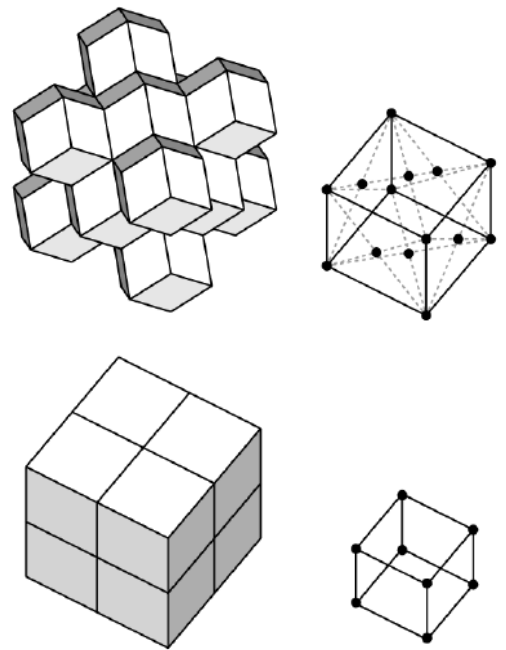

Figure 6 Examples of Combination of Convex Polyhedrons in Crystal Structures

Quasicrystal structure does not own the attribute of translational symmetry, but can repeats in certain rules in various positions and directions, following Bravais laws
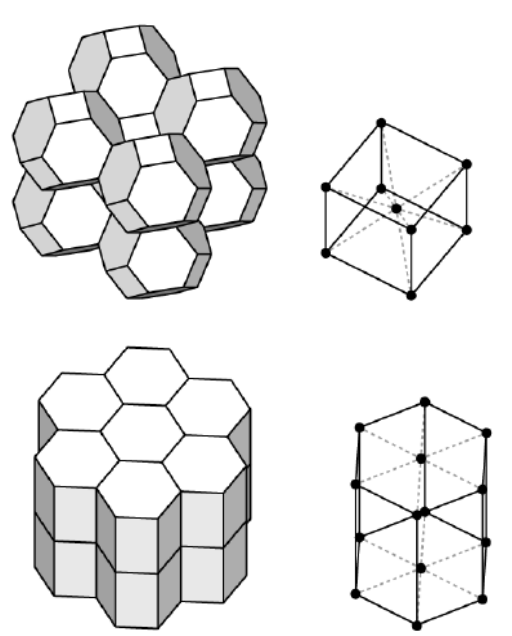

Similar with two-dimensional tessellations, translational symmetry is still the basis criterion of periodicity for three-dimensional tessellations. Much attention has been focused on the field of crystal morphology in the study of three-dimensional periodic tessellation since crystals have internal structures arranged according to certain geometric rules, while internal structures of non-crystals are arranged without regular rules or even randomly. Therefore, the internal structure of crystal can be regarded as a periodic spatial tessellation, which can fill the space closely by convex polyhedrons of the same shape and size in ideal conditions.

to generate geometric polyhedrons spontaneously. For example, Rhombic Hexecontahedron can be composed of 20 long rhombohedral bodies, while Rhombic 
triacontahedron can be composed of 10 "long rhombus" and 10 "flat rhombus". Combination of these two polyhedrons generates on typical aperiodic threedimensional tessellation.

\subsubsection{Other Types of Three-Dimensional Tessellation (E.G.)}
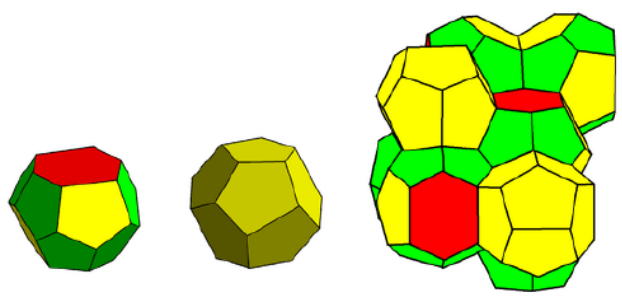

Figure 7 WP Structure Unit triangles. Each tetrahedron can be split into another Danzer Tiling with smaller proportions tetrahedron mosaic, and its expansion rate is exactly the golden number $\pi$. Different combinations of these tetrahedra can form a variety of rotationally symmetric threedimensional aperiodic tessellation.
The Danzer Tiling consists of four different tetrahedra A, $\mathrm{B}, \mathrm{C}$, and $\mathrm{K}$, and the unfolded diagrams of which can be regarded as the different arrangements of 4 different

\subsubsection{WP structure}

The WP structure consists of two irregular polyhedrons of equal size but of different shapes. This tessellation gives great answers to the famous question: how to divide space into equal volume units with the most economical structure segmentation and the least amount of interface areas.
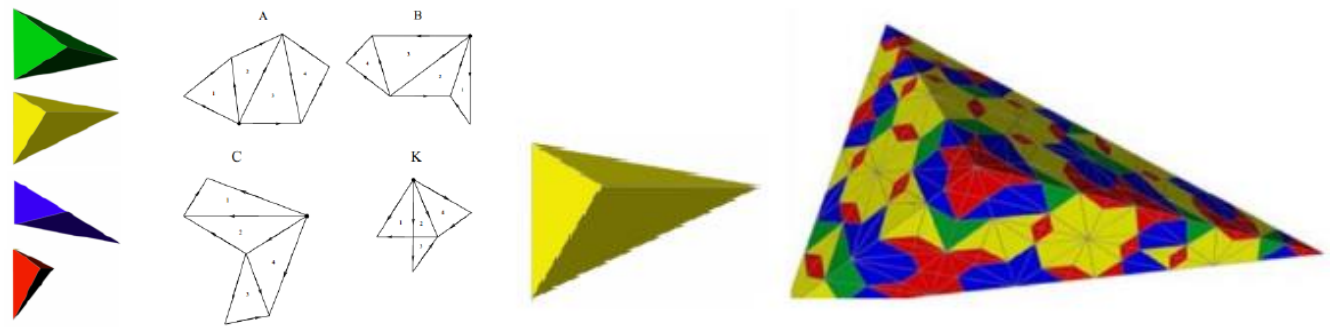

Figure 8 Tetrahedra and Expansion Rate in Danzer Tiling

\section{The Application of Tessellation in Architectural Geometry Design}

\subsection{Application of Tessellation in Architecture Through History}

Application of tessellation in architecture can be dated back to the beginning of civilization. It is widely found in many civilizations of the world, including ancient Egypt, Moors, Romans, Persia, Greece, Byzantium, Arabia,
Japan and China, existing throughout the history of architecture development.

The ancient Romans liked to use mosaics, as one of the most common tessellations to decorate their floors and ceilings. These decorations varied according to different spaces inside the building. This style also influenced Islamic architecture greatly, using ceramics, marble, glass, glazed pottery to complete mosaic decoration. While in Gothic cathedral, radial tessellation was designed as rose windows, and arrangement of arch and its complicated structures were also typical examples of application of tessellation in architectural design.

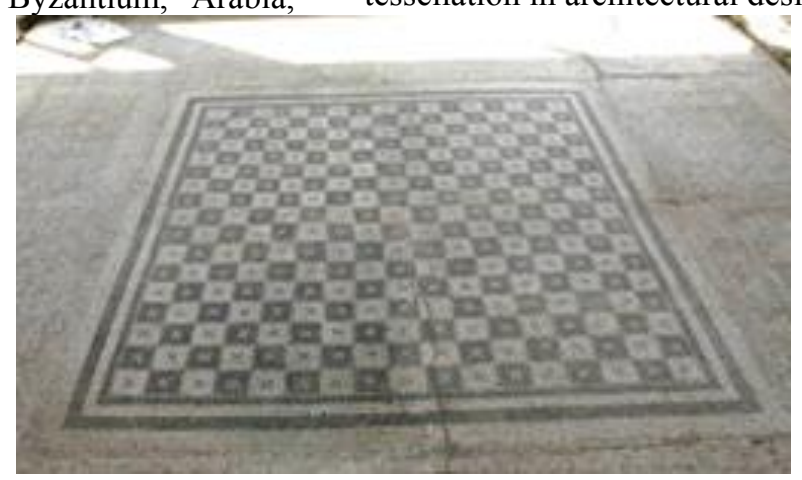

Figure 9 Ground Floor Pavement of Rome Villa in Italy 


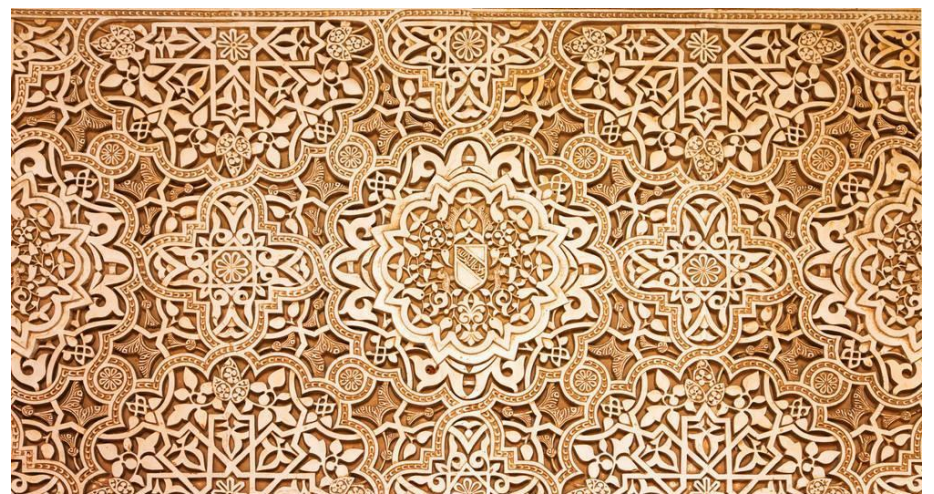

Figure 10 Tessellation in Alhambra Palace

In Chinese traditional architecture, flower windows and floor tiles are often decorated with geometric patterns to form regular, rhythmic tessellations.
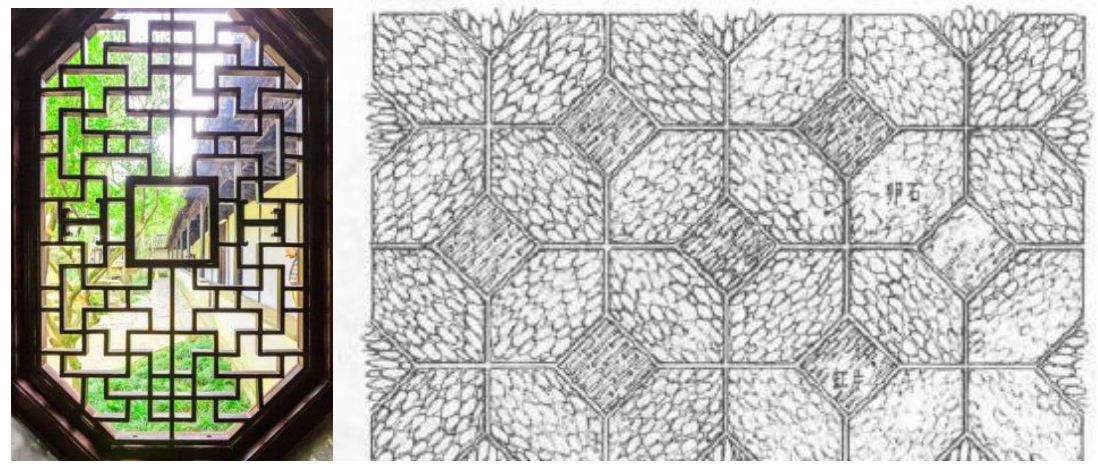

Figure 11 Tessellations in Windows and Floors in Chinese Traditional Gardens.

\subsection{Application of Tessellation in Modern Architecture}

Corbusier' promotion of modular design after his theoretical research and analysis of human body and its related mathematics bought in new generation of modern architecture. Standard modular system in architecture is encouraged world-widely, including basic modulus and enlarged modulus. It is a three-dimensional modular grid structure of certain rules, which can be regarded as one type of three-dimensional tessellations. Therefore, this tessellation provides the basis for the form, structure and space design of the building.

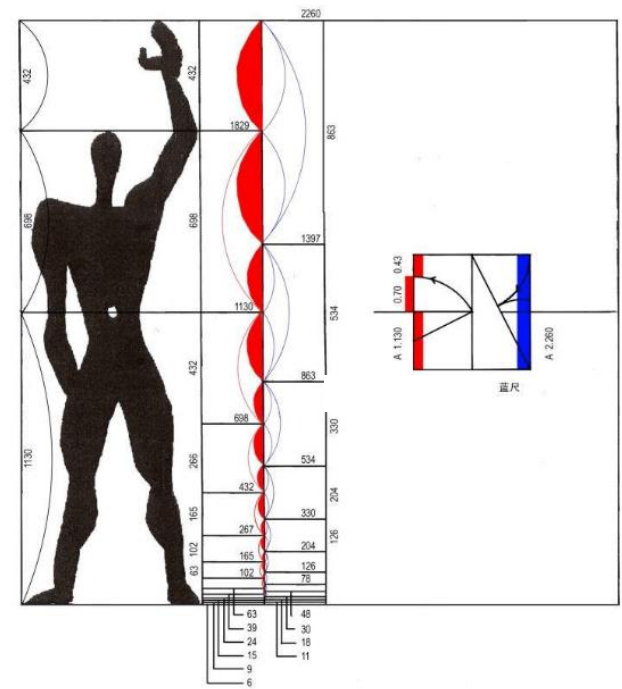

Figure 12 Human Body Modular Analysis by Corbusier

\subsection{Application of Tessellation in Computer Geometric Technology}

Geometric tessellation is one of the foundations of computer graphics. The application of geometric tessellation in computer technology is based on the 
algorithm of surface, from the input of the surface to the output of the geometry.

\subsubsection{Surface Control in Modeling}

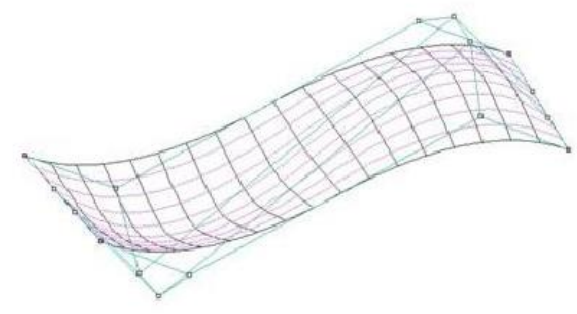

Computer surface modeling can use a variety of methods, such as NURBS curve mesh modeling, mesh (Polygon Mesh) modeling, polygon mesh subdivision modeling (Subdiv). All these methods need the control and editing of the surface mesh, which can never be achieved without the application of tessellation.

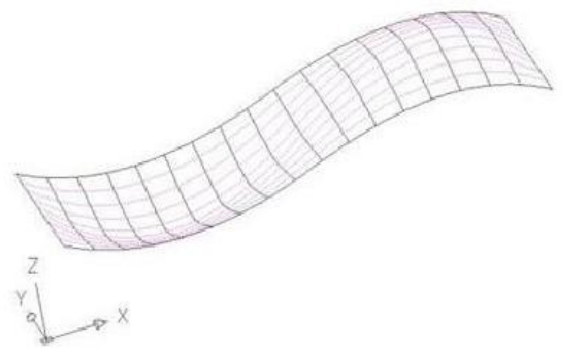

Figure 13 NURBS Control

\subsubsection{Subdivision of Surfaces in Display}

In the aspect of computer graphics display, surface subdivision technology is the technology of managing graphic data by embedding graphics, and subdividing the graph to make it easy to render. It is called "mosaic (one classical tessellation) processing technology". Computers can not directly generate curves, nor can they directly generate surfaces. The surface displayed on a computer screen is actually made up of a number of dots of polygonal patches. Computer surface subdivision is to refine the original rough mesh model by using proper topologies and geometric rules, so as to generate smooth surfaces.

\subsection{0-14 Tower}

The 0-14 Tower in Dubai was designed by Reiser + Umemoto in 2007. Rather than a glass curtain wall which is visible everywhere in Dubai, the facade uses concrete pouring to form a shell structure. Large and small circular windows composed its unique facade. These windows were generated accoding to various factors based on rectangular grid. Approximate circular window arrays were formed by inner offset and chamfer of each unit in the oblique rectangular grid. The amount of offseting was related with parameters such as indoor lighting conditions and structure forces. Moreover, the mutation of several units in the oblique rectangle grid provides better outside views for the inside.

\section{Modern Cases}

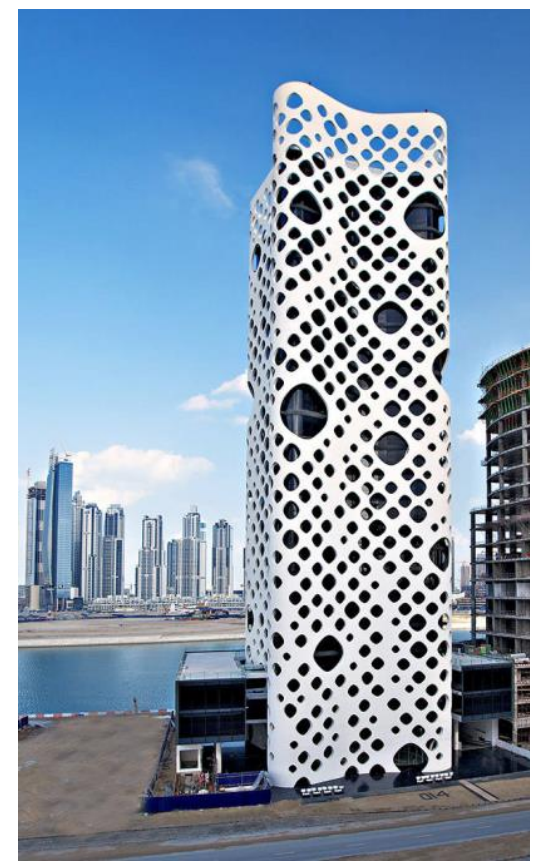

Figure 14 0-14 Tower and Its Skin Design Process

Designed by CSCEC, CCDI, PTW and ARUP, the steel structure of the "Water Cube" (National Aquatics Center
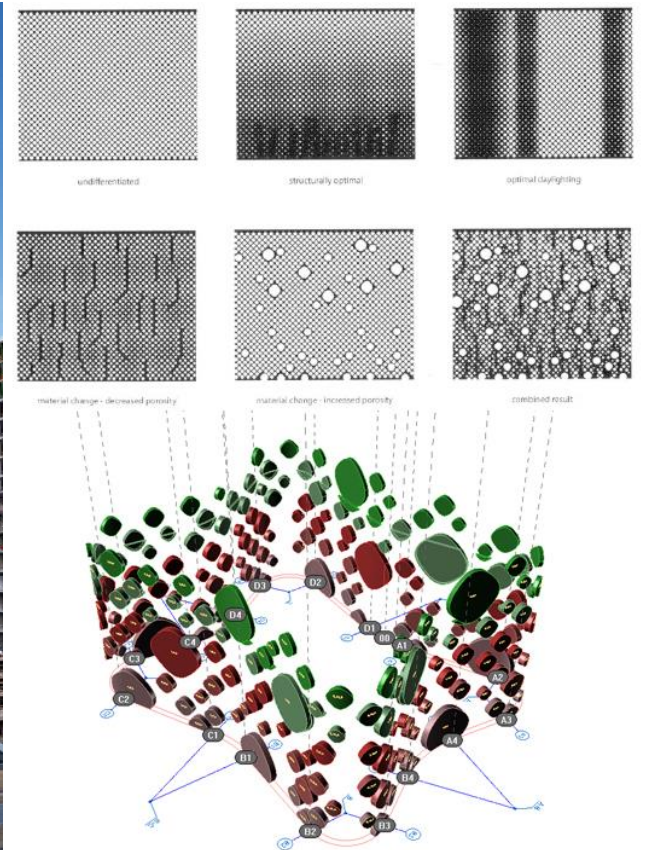
of the Beijing Olympic Games) is a three-dimensional tessellation structure made of WP units. In the design process WP units were firstly used to produce a tessellation structure construction larger than the volume of "Water Cube", after which the structure was rotated by 60 degrees based on vector axis, from which a $176.5 \mathrm{~m} \mathrm{x}$ $176.5 \mathrm{~m}$ x $29.4 \mathrm{~m}$ cubic was cutting out as the building's gerometry. Spaces of different funtions were later generated from inside, thus forming a spatial grid steel structure. Since the cutting direction is not parallel or orthogonal to its translation direction, the facade is not smooth. However, in fact it still keeps the translational periodicity as WP tessellation does.
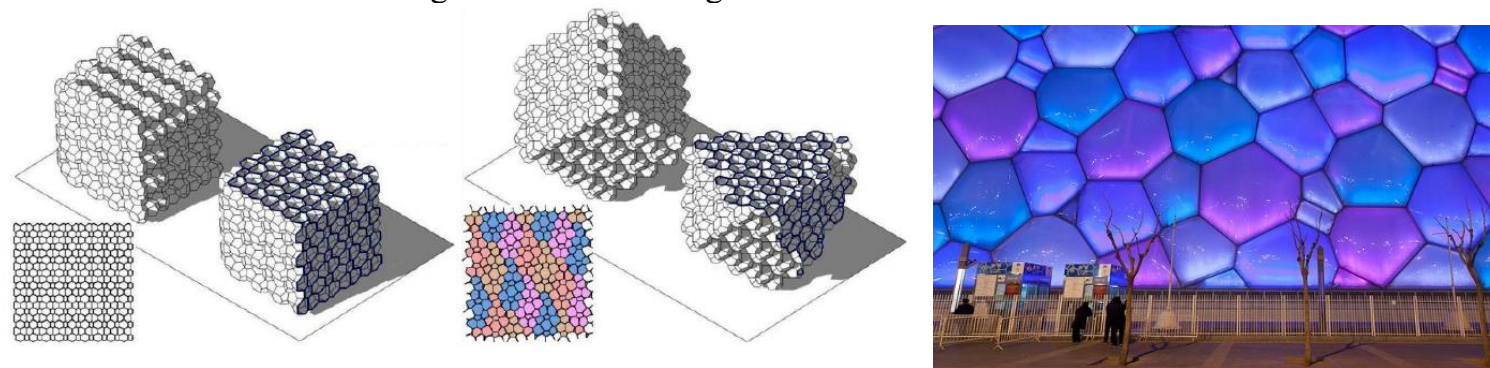

Figure 15 0-14 Water Cube and Its Structure Analysis

\subsection{Hybrid Bio Structures}

Hybrid Bio Structures was designed by Riyad Joucka in his master's degree project in AA (U.K.). This project revealed another application of Voronoi tessellation. Voronoi tessellation get features of "field", so in the conditon of membrane structure with a plurality of support points, the grid generated through Voronoi algorithm reflected the gravity of maximal deformation in the membrane structure. Voronoi tessellation was generated after setting "seeds" (based on indoor function, lighting environment, etc.) positions. Roof of complex membrane structure was formed through point stretching, smoothing and other movements of the units. This architecture is a pavalion with multi-functions, and the Voronoi grid remained through the tour route for visitors.
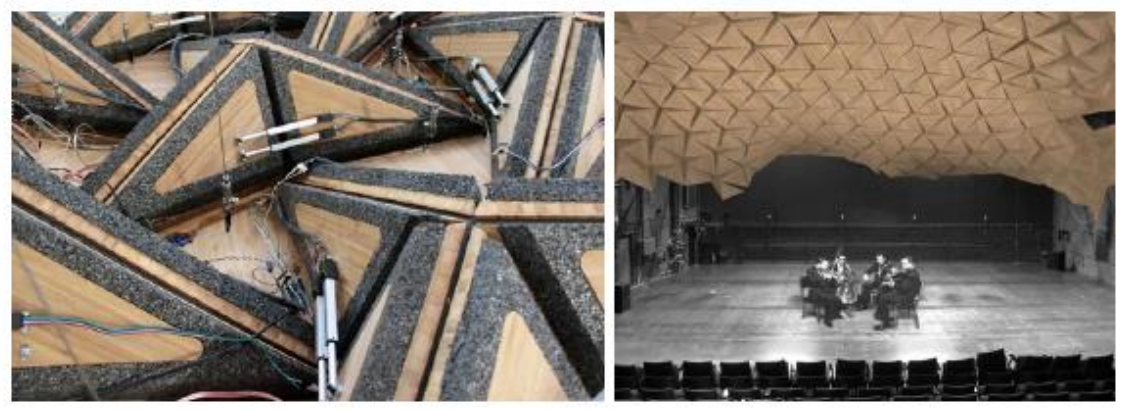

Figure 16 The Component Unit of Hybrid Biostructure

\section{Conclusion}

As significant influential factor of architecture, tessellation runs through the whole architecture history of the East and the West. At present, it is also the basis of graphic technologies in the development of digital technology. It acts as a link between architectural design and digital technology, widely used in contemporary architectural design, especially architectural geometry design.

Since tessellation geometry is usually a combination of homogeneous units, it has outstanding advantages in building skin, construction ability, structure property and so on. In addition, standing at the source position in the computer surface technology, tessellation contributes to the easy set-up and control of surface design in architectural geometry. With the application of tessellation, architectural geometry can be more flexible and contains various forms of different compositions, being more adopted in dealing with different problems. Therefore, such application in architectural geometry design will be of great benefit and potential with thoughtful consideration.

\section{References}

1. Branko Grunbaum, Tilings and Patterns[M]. W.H. Freeman, 1987.

2. Lynn G. Animate Form[M]. Princeton Architectural Press, 1999.

3. Farshid Moussavi, The Function Of Form[M]. Harvard University Graduate School of Design,2009.

4. Kostas Terzidis, Algorithmic Architecture[M]. Architectural Press, 2006.

5. Michael Meredith, From Control to Design: Parametric/Algorithmic Architecture[M]. Actar/Barcelona Regional, 2008.

6. Paul Jackson, Folding Techniques for Designers: From Sheet to Form[M]. Laurence King, 2011.

7. Eric Gjerde, Origami Tessellations Awe-Inspiring 
Geometric Designs[M]. A K Peters,2008.

8. Michael Fox, Interactive Architecture[M]. Princeton Architectural Press, 2009

9. Jingzhong Chen, Basic properties of quasicrystals[J]. Proceedings of China University of Geosciences, 1993-S1.

10. Xianguang Chen, Study on the Shape Finding of Tensegrity Units [J]. Space Structure, 2003-01.

11. Robert W.Fathauer, Fractal tilings based on v-shaped prototiles[J]. Computers \& Graphics, 2002.

12. Danzer L., Three-dimensional analogs of the planar penrose tilings and quasicrystals[J]. Discrete Mathematics, 1989. 